\title{
The metabolic syndrome modifies
} the mRNA expression profile of extracellular vesicles derived from porcine mesenchymal stem cells

Yu Meng ${ }^{1,5}$, Alfonso Eirin ${ }^{1}$, Xiang-Yang Zhu' ${ }^{1}$, Daniel R. O'Brien², Amir Lerman ${ }^{3}$, Andre J. van Wijnen ${ }^{4}$ and Lilach O. Lerman ${ }^{1,3^{*}}$

\begin{abstract}
Background: Mesenchymal stem cells (MSCs) perform paracrine functions by releasing extracellular vesicles (EVs) containing microRNA, mRNA, and proteins. We investigated the mRNA content of EVs in metabolic syndrome (MetS) and tested hypothesis that comorbidities interfere with the paracrine functionality of MSCs.

Methods: Mesenchymal stem cells were collected from swine abdominal adipose tissue after 16 weeks of a low(Lean) or high-calorie (MetS) diet ( $n=5$ each). We used next-generation mRNAs sequencing to identify mRNAs enriched and depleted in Lean- or MetS-EVs compared to the parent MSCS.

Results: We found 88 and 130 mRNAs enriched in Lean-EVs and MetS-EVs, respectively, of which only eight were common genes encoding proteins related to the nucleus, endoplasmic reticulum, and membrane fraction. Lean-EVs were enriched with mRNAs primarily involved in transcription regulation and the transforming growth factor (TGF) $\beta$ signaling pathway, but devoid of genes related to regulation of inflammation. In contrast, MetS-EVs contained mRNAs involved in translational regulation and modulation of inflammation mediated by chemokines and cytokines, but lacked mRNAs related to TGF- $\beta$ signaling. mRNAs enriched in EVs have the potential to target a significant proportion of genes enriched in EVs, but only $4 \%$ microRNA target genes overlap between Lean- and MetS-EVs. Co-culture with MetS-EVs also increased renal tubular cell inflammation in-vitro.
\end{abstract}

Conclusions: Metabolic syndrome may affect immunomodulatory function of porcine MSCs by modifying mRNA profiles of the EVs that they produce and post-transcriptional regulation. These observations may have important implications for cell-based therapy, and support development of strategies to improve the efficacy of MSCs and their EVs.

Keywords: Mesenchymal stem cells, Extracellular vesicles, mRNA, Metabolic syndrome, Swine

\footnotetext{
*Correspondence: Lerman.Lilach@Mayo.edu

${ }^{1}$ Divisions of Nephrology and Hypertension, Mayo Clinic, 200 First Street

SW, Rochester, MN 55905, USA

Full list of author information is available at the end of the article
} 


\section{Background}

Mesenchymal stem/stromal cells (MSCs) are non-embryonic stem cells that have the potential to proliferate and differentiate into several cell types, and can be isolated from the umbilical cord, bone marrow, adipose tissue, and other tissues [1]. Because MSCs possess important pro-angiogenic, immunomodulatory, and anti-inflammatory properties, they are a promising source for cellbased strategies that support tissue regeneration [2]. Indeed, over 200 clinical trials are currently testing the efficacy of MSC therapy for treating a variety of diseases [3].

MSCs exert their reparative actions in part by releasing extracellular vesicles (EVs), membrane microparticles including microvesicles and exosomes that shuttle their genetic and protein cargo to damaged tissues $[4,5]$. Indeed, studies using a pig model of renal vascular disease have shown that EVs may activate an endogenous repair program in parenchymal cells $[6,7]$. We have previously shown that adipose tissue-derived MSCs release EVs that transport gene and protein-based regulatory information to modulate angiogenesis, apoptosis, extracellular matrix remodeling and other cellular pathways in recipient cells [8-10], underscoring the reparative potential of MSCs. For example, MSC-derived EVs are selectively enriched with mRNAs encoding for transcription factors, Golgi apparatus components, and proteins involved in transforming growth factor (TGF)- $\beta$ signaling. However, comorbidities and cardiovascular risk factors that alter the microenvironment from which these cells are harvested may potentially impact on the cargo packed within their EV progeny [11-13].

The metabolic syndrome (MetS) is a constellation of cardiovascular risk factors, including obesity, hypertension, dyslipidemia, increased serum triglycerides, and insulin resistance, that increases cardiovascular morbidity and mortality. In a recently developed porcine model of MetS and fat inflammation [14], we found that adipose tissue-derived MSCs show increased propensity for adipogenesis and for premature senescence compared to Lean-MSCs [15]. In the current study, we addressed the key question whether this phenotypic change is accompanied by altered packaging of mRNA cargo in their EV paracrine vectors. We tested the hypothesis that MetS affects the phenotype of MSCs, which in turn modifies the mRNA expression profile in EVs derived from MSCs. Expression profiles of mRNAs were obtained in EVs and their parent MSCs, using high-throughput RNA sequencing (RNA-seq). We show that the mRNA cargo of MetS-EVs is characterized by genes primarily involved in inflammation. Yet, mRNAs enriched in Lean-EVs are involved in tissue repair and TGF- $\beta$ signaling, and these mRNAs appear to be excluded from MetS-EVs. Lastly,
microRNAs enriched in EVs have the potential to target a significant proportion of genes enriched in Lean and MetS EVs, implying post-transcriptional regulatory mechanisms. These observations may help refine cellbased therapy and support development of adequate strategies to improve the efficacy of MSCs and their EVs.

\section{Methods \\ Experimental design}

Animals were studied with the approval of the Institutional Animal Care and Use Committee. Three-monthold female domestic pigs were randomized as MetS or Lean $(\mathrm{n}=5$ each). Lean pigs were fed with standard chow (13\% protein, 2\% fat, 6\% fiber, Purina Animal Nutrition LLC, MN), and MetS pigs with a high-cholesterol/carbohydrate diet $(5 \mathrm{~B} 4 \mathrm{~L}$, protein $16.1 \%$, ether extract fat $43.0 \%$, and carbohydrates $40.8 \%$, Purina Test Diet, Richmond, IN) [14] for a total of 16 weeks. All animals had free access to water.

Body weight and blood pressure (using an intra-arterial catheter) were recorded after 16 weeks of diet. Total cholesterol, low-density lipoprotein (LDL), triglyceride, fasting glucose, and fasting insulin levels were measured by enzymatic methods. Insulin resistance was assessed by the homeostasis model assessment of insulin resistance (HOMA-IR). After completion of all studies, animals were euthanized with a lethal intravenous dose of sodium pentobarbital $\left(100 \mathrm{mg} / \mathrm{kg}\right.$ IV; Sleepaway ${ }^{\circledR}$; Fort Dodge Laboratories, Fort Dodge, IA, https://www.zoeti sus.com), and subcutaneous abdominal adipose tissue collected for MSC and EV isolation.

\section{MSC and EV isolation, characterization, and culture}

Mesenchymal stem cells were isolated from swine subcutaneous abdominal fat tissue, which was digested in collagenase- $\mathrm{H}$, filtered, and cultured for 3 weeks in advanced MEM medium (Gibco/Invitrogen) supplemented with $5 \%$ platelet lysate, as previously described [10]. At passage 3, MSCs were collected and cellular phenotype confirmed by the expression of the MSCs markers CD73, CD105, CD44, and CD90, as well as by their capacity for tri-lineage differentiation, as previously shown $[16,17]$.

Extracellular vesicles were isolated from passage 3-MSC supernatants $\left(10 \times 10^{6}\right.$ cells $)$ by ultracentrifugation, as previously described [8-10, 18, 19]. Samples were centrifuged ( $2000 \mathrm{~g}$ for $20 \mathrm{~min}$ ), and the supernatant collected and subsequently centrifuged $(100,000 \mathrm{~g}$ for $1 \mathrm{~h}$ ) at $4{ }^{\circ} \mathrm{C}$. EVs were collected, suspended in wash buffer medium 199, centrifuged once more $(100,000 \mathrm{~g}$ for $1 \mathrm{~h})$, and characterized based on the expression of common EV (CD9 and CD63) and MSC surface markers, as previously shown $[8,10,20]$. 


\section{Sequencing of mRNA and data analysis}

MSC and EV mRNA sequencing was performed as described $[8,18]$ and data analyzed using the MAPRSeq v1.3 workflow [21, 22]. EdgeR2.6.2 [23] was used to analyze the differential expression to identify mRNAs enriched in Lean- and MetS-EVs compared to their parent MSCs. Expression values for each gene were normalized by the total number of reads per sample and corrected for gene length (reads per kilobasepair per million mapped reads, RPKM) (Additional File 1). Genes with RPKM $>0.1$, fold-change (EVs/MSCs) $>1.4$, and $\mathrm{p}<0.05$ (EVs vs. MSCs, Student's t-test) were classified as enriched in EVs [24]. Conversely, we considered genes with $\mathrm{RPKM}>0.1$, fold-change (EVs/MSCs) $<0.7$, and $\mathrm{p}<0.05$ to be excluded from EVs. Functional annotation clustering and pathway analysis were performed using DAVID 6.8 database (https://david-d.ncifcrf.gov/) and PANTHER Classification System (http://pantherdb.org/) $[8,10,18]$.

To elucidate whether mRNAs enriched in MSCderived EVs are implicated in insulin signaling, we used the GeneCards ${ }^{\circledR}$ database (http://www.genecards.org/) to screen genes associated with insulin signaling [25].

\section{Validation of mRNA sequencing analysis}

For validation, we randomly selected the mRNAs ANP32B, ARHGAP11B, CCL27, CLDN22, NR5A1, HIST1H2AJ, and PTX3, which were dysregulated in Lean and MetS-MSCs versus their daughter EVs, and measured their expression by quantitative polymerase chain reaction (qPCR) with GAPDH as reference gene. Total RNA was isolated from $5 \times 10^{5}-1 \times 10^{6} \mathrm{MSC}$ and EV samples. All primers were from ThermoFisher Scientific (Catalog Numbers: ANP32B: AP322J4, ARHGAP11B: APXGRAH, CCL27: ss03378478, CLDN22: ss04321456, NR5A1: ss03394295, HIST1H2AJ: AP2W7Y7, and PTX3: ss04328596).

\section{Interactions among microRNAs and mRNAs enriched in EVs} We have recently shown that Lean- and MetS-EVs were enriched in, respectively, 14 and 8 distinct microRNAs [26]. Hence, we sought to identify target genes for these microRNAs among the mRNAs packed within the same EVs. Venn diagrams were therefore constructed using VENNY 2.1 (http://bioinfogp.cnb.csic.es/tools/venny/) to visualize common miRNA target genes enriched in Leanand MetS-EVs.

\section{MSC-derived EV effects on inflammation}

Human monocytes were cultured for $18 \mathrm{~h}$ in RPMI 1640 media supplemented with M-CSF, LPS, and IF- $\gamma$ to induce M1 polarization [27]. M1-polarized cells were cultured alone or co-cultured with Lean- and MetSMSC-derived EVs (50 $\mu \mathrm{g}$ of EV protein). Expression of inducible nitric oxide synthase (iNOS) and arginase-1 (1:200, Santa Cruz, CA) was evaluated using Western blotting [17]. In addition, pig proximal kidney tubular epithelial cells (LLC-PK1, ATCC, Manassas) were cultured in Medium-199 (Gibco BRL, USA) containing 3\% FBS [28] alone or co-cultured with Lean- and MetSMSC-derived EVs (5 $\mu$ g of EV protein). Tubular epithelial cell inflammation was evaluated by immunofluorescent staining with antibodies against tumor necrosis factor (TNF)- $\alpha$ (Santa Cruz, 1:200) and monocyte chemoattractant protein (MCP)-1 (MyBioSource, San Diego, CA, http://www.mybiosource.com 1:7500).

\section{Statistical analysis}

Statistical analysis was performed using JMP 13.0 (SAS Institute, Cary, NC, USA). Data were expressed as mean \pm standard deviation. Student's t-test was used to evaluate significant differences between the Lean and MetS groups. Statistical significance was accepted if $\mathrm{p} \leq 0.05$.

\section{Results}

Systemic characteristics

The systemic characteristics of all animals at 16 weeks are shown in Table 1. Body weight and blood pressure were higher in MetS pigs compared to Lean. Fasting glucose levels were comparable, but other MetS indices like insulin level and HOMA-IR score were higher in MetS vs. Lean, as were total cholesterol, LDL, and triglyceride levels. These findings imply development of MetS in our pig model.

\section{Genes enriched in Lean- and MetS-EVs}

Of all annotated genes $(n=16,656)$, mapping of RNA reads revealed 88 mRNAs upregulated in Lean-EVs compared to Lean-MSCs (Fig. 1a, b), and 130 mRNAs enriched in MetS-EVs compared to their parent

Table 1 Systemic characteristics in the experimental pig groups ( $n=5$ each) at 16 weeks

\begin{tabular}{lccc}
\hline Parameter & Lean & MetS & p value \\
\hline Body weight $(\mathrm{kg})$ & $69.3 \pm 11.4$ & $92.2 \pm 4.4^{*}$ & 0.0005 \\
Mean blood pressure $(\mathrm{mmHg})$ & $97.9 \pm 13.1$ & $124.1 \pm 5.7^{*}$ & 0.002 \\
Total cholesterol $(\mathrm{mg} / \mathrm{dL})$ & $79.5 \pm 7.3$ & $354.2 \pm 30.5^{*}$ & $<0.0001$ \\
LDL cholesterol $(\mathrm{mg} / \mathrm{dL})$ & $31.7 \pm 6.9$ & $349.0 \pm 132.1^{*}$ & 0.0003 \\
Triglycerides $(\mathrm{mg} / \mathrm{dL})$ & $6.8 \pm 1.7$ & $17.1 \pm 2.4^{*}$ & 0.0002 \\
Fasting glucose $(\mathrm{mg} / \mathrm{dL})$ & $108.8 \pm 23.1$ & $123.7 \pm 22.3$ & 0.207 \\
Fasting insulin $(\mu \mathrm{U} / \mathrm{mL})$ & $0.4 \pm 0.1$ & $0.7 \pm 0.1^{*}$ & 0.002 \\
HOMA-IR score & $0.6 \pm 0.1$ & $1.7 \pm 0.3^{*}$ & $<0.0001$ \\
\hline
\end{tabular}

HOMA homeostasis model assessment of insulin resistance, $L D L$ low-density lipoprotein, Mets metabolic syndrome

${ }^{*} \mathrm{p} \leq 0.05$ vs. Lean 


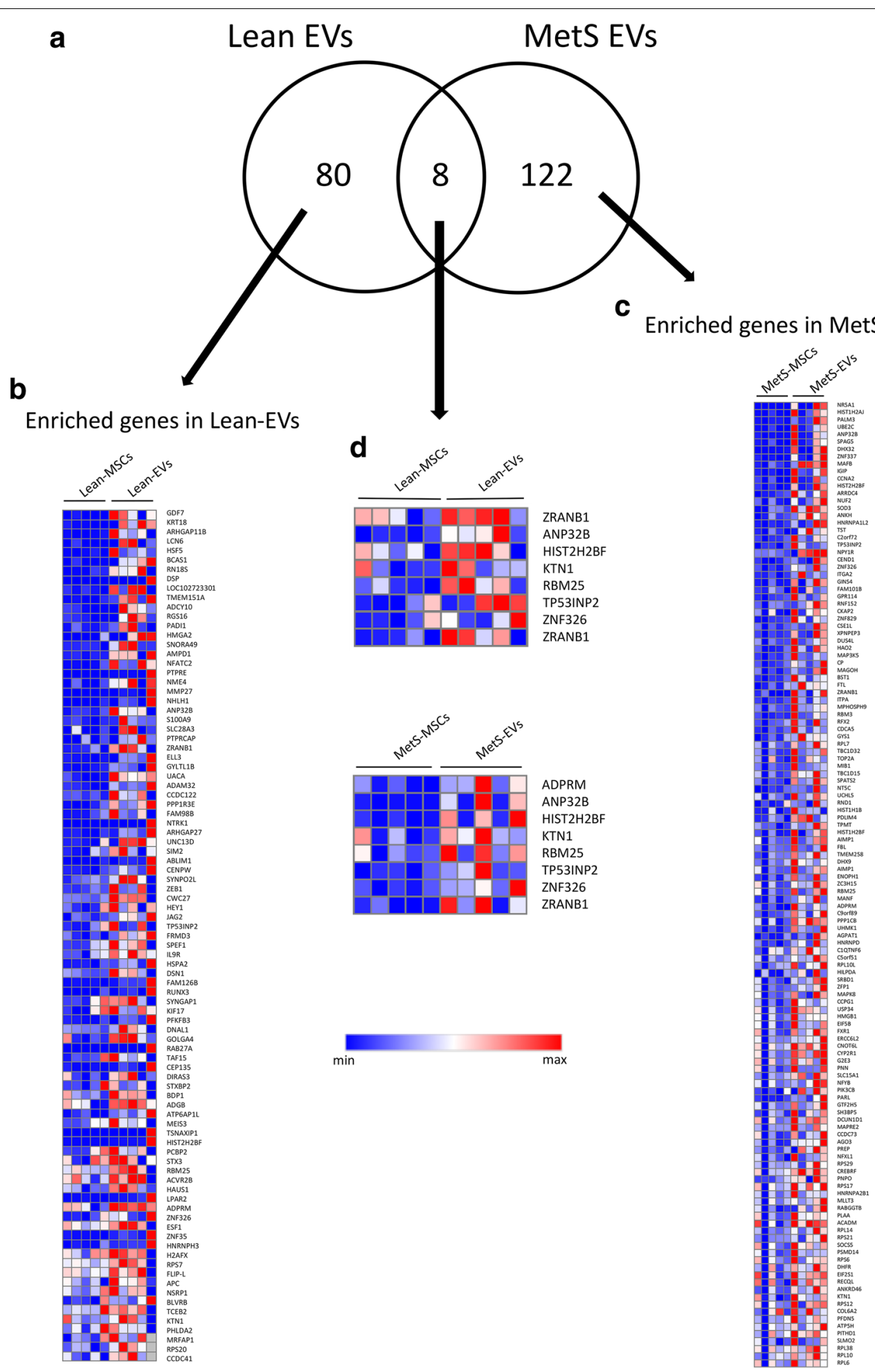

Fig. 1 Enrichment of mRNAs in Lean- and MetS-EVs. a Of 16,656 annotated genes, mapping of RNA reads revealed 88 mRNAs enriched in Lean-EVs and 130 in MetS-EVs compared to their parent MSCs, with only eight common mRNAs. b Heat map of mRNAs enriched in Lean-EVs. $\mathbf{c}$ Heat map of mRNAs enriched in MetS-EVs. $\mathbf{d}$ Heat map of enriched genes shared between the Lean-EVs and MetS-EVs 
MetS-MSCs (Fig. 1a, c). Of those, only eight enriched genes were shared between the Lean-EVs and MetS-EVs (Fig. 1a, d), and were found to encode proteins related to the nucleus, endoplasmic reticulum, and membrane fraction.

Functional annotation analysis showed that Lean-EVs selectively contain genes encoding regulators of transcription (Fig. 2a), including (Fig. 1b) meishomeobox-3 (MEIS3), hairy/enhancer-of-split related with YRPW motif 1 (HEY1), and heat-shock transcription factor family member 5 (HSF5). Biological pathway analysis showed that genes enriched in Lean-EVs encode proteins involved in 50 pathways. Overall, Lean-EVs enriched genes were involved in isopeptide bond, non-membrane-bounded organelle, and vesicle-mediated transport. Genes enriched in Lean-EVs also encode proteins involved in axon guidance mediated by netrin (P00009), angiogenesis (P00005), Wnt signaling pathway (P00057), and TGF- $\beta$ signaling pathway (P00052) (Fig. 2c), confirming our previous observations in healthy pigs [8].

Contrarily, genes enriched in MetS-EVs encode proteins involved in translation elongation and ribonucleoprotein complex (Fig. 2b), including (Fig. 1c) eukaryotic translation initiation factor-2 subunit-alpha (EIF2S1), eukaryotic translation initiation factor 5B (EIF5B), ribosomal protein S17 (RPS17), and ribosomal protein (RP)L6, $7,10,14$. In contrast to Lean-EVs, mRNAs enriched in MetS-EVs are linked to 25 pathways and encode proteins involved in integrin signaling (P00034), inflammation mediated by chemokine and cytokine signaling (P00031), ubiquitin-proteasome (P00060), and fibroblast growth factor (FGF) signaling (P00021) pathways (Fig. 2d). Gonadotropin-releasing hormone receptor (P06664) and apoptosis signaling (P00006) were among the top six pathways regulated by genes enriched in both Lean- and MetS-EVs (Fig. 2c, d).

Twenty-one mRNAs related to insulin signaling were enriched in Lean-EVs (Table 2), whereas 19 distinct genes were enriched in MetS-EVs (Table 3). Two mRNAs enriched in MetS-EVs (NR5A1 and SLC15A1) were also enriched in MetS-MSCs, as we observed in a recent previous study [25].

\section{Genes excluded from Lean- and MetS-EVs}

A total of 4166 mRNAs were excluded from Lean-EVs and 2449 from MetS-EVs. Annotation analysis of the top 100 mRNAs (filtered by fold-change EVs/MSCs) excluded from Lean- and MetS-EVs showed only nine common genes (Fig. 3a), which were involved in lipase activity, ion channel activity, oxidoreductase activity, and extracellular matrix structural constituents. LeanEVs lacked inflammatory genes (23\% of top 100 mRNAs excluded from Lean-EVs), such as those involved in regulation of leukocyte proliferation, immunoglobulin secretion, and B-cell activation (Fig. 3b). 12\% of the top 100 mRNAs selectively excluded from MetS-EVs were related to TGF- $\beta$ signaling, a pathway that seems to be blunted in MetS. MetS-EVs also lacked mRNAs related to positive regulation of RNA metabolic processes, positive regulation of transcription, carboxylic acid biosynthetic process, cellular cation homeostasis, and contractile fiber part (Fig. 3c).

\section{Validation of RNAseq findings}

PCR-determined expression of the random candidate mRNAs confirmed the same pattern detected by mRNAseq. Specifically, ANP32B and ARHGAP11B were higher, and CCL27 and CLDN22 lower in Lean-EVs compared to their parent MSCs. Expression of NR5A1 and HIST1H2AJ was higher, but expression of PTX3 was lower in MetS-EVs vs. MetS-MSCs (Fig. 4).

\section{mRNA-microRNA interaction analysis}

We took the opportunity to seek among the genes identified in the current study, targets for microRNA that we observed in a recent previous study. Interestingly, we found that those microRNAs enriched in EVs [18] have the potential to target a significant proportion of genes enriched in Lean and MetS-EVs (50 and 84, respectively), but only $5(3.9 \%)$ of microRNA target genes overlap between Lean- and MetS-EVs (Fig. 5).

\section{Effects of MSC on inflammation}

Co-culture with either Lean- or MetS-MSC-derived EVs similarly decreased the expression of iNOS and increased the expression of arginase- 1 compared to untreated M1-polarized macrophages (Fig. 6). However, when cocultured with renal tubular cells, MetS-EVs induced a greater expression of TNF- $\alpha$ and MCP-1 compared to untreated cells and cells co-cultured with Lean-EVs (Fig. 7).

\section{Discussion}

Our study employed high-throughput mRNA sequencing to interrogate mRNA expression in EVs and their parent MSCs, and explore the putative function of enriched or excluded genes in Lean- vs. MetS-EVs. The current study demonstrates that MetS modulates the mRNA cargo packed within porcine adipose tissue MSC-derived EVs, as the mRNA content of Leanand MetS-EVs is substantially different. Lean-EVs are enriched with mRNAs primarily involved in transcription regulation and TGF- $\beta$ signaling pathway, but not in genes related to regulation of inflammation. In contrast, MetS-EVs contain mRNAs involved in translational regulation and modulation of inflammation, but 
a

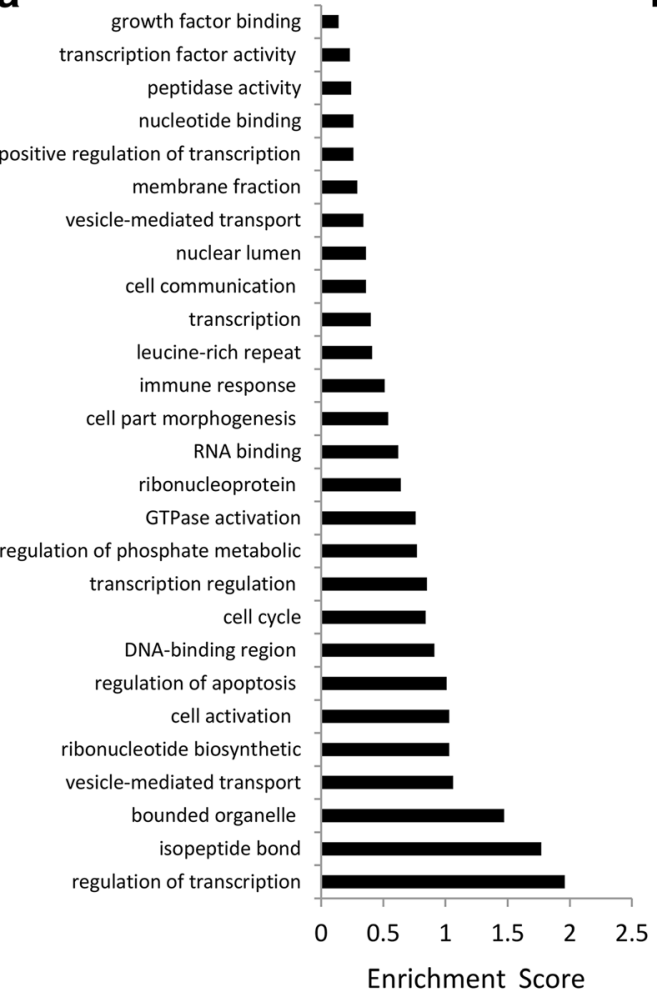

C

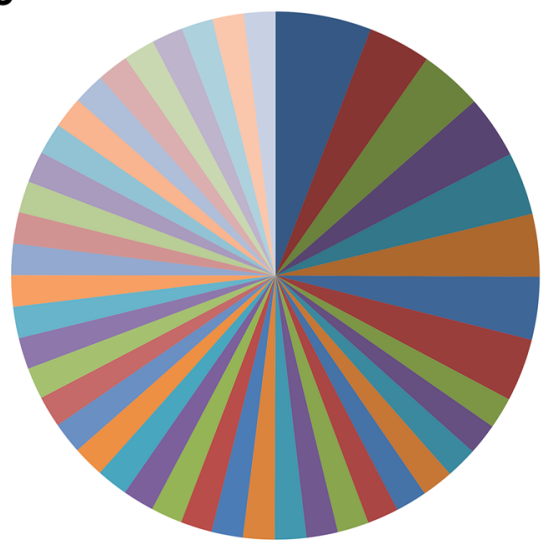

- Gonadotropin-releasing hormone receptor pathway (P06664)

- Axon guidance mediated by netrin (P00009)

- Apoptosis signaling pathway (P00006)

- Angiogenesis (P00005)

n Wnt signaling pathway (P00057)

n TGF-beta signaling pathway (P00052) b

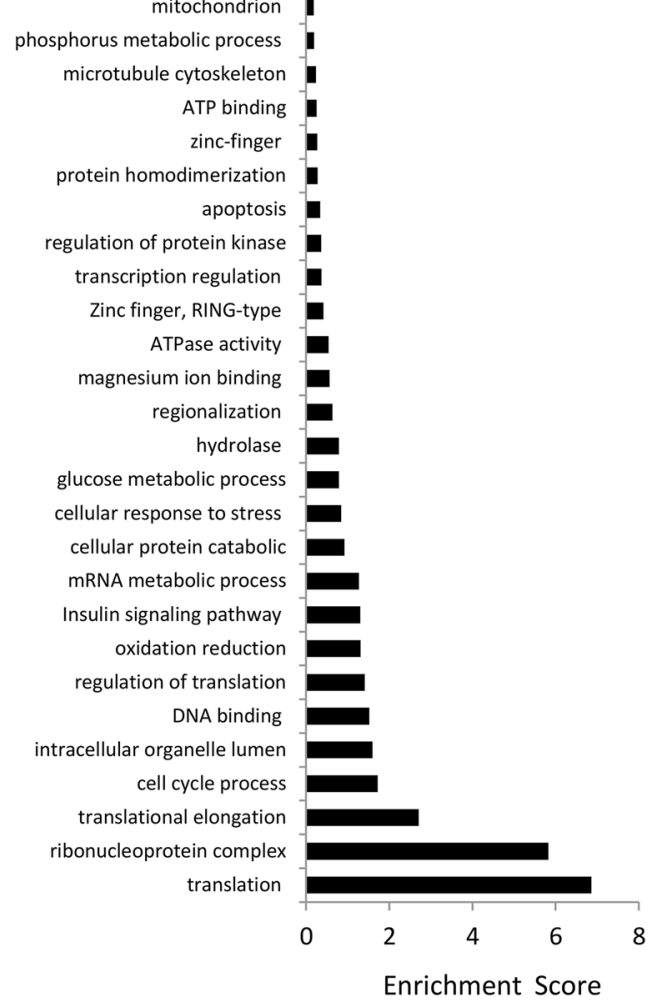

d

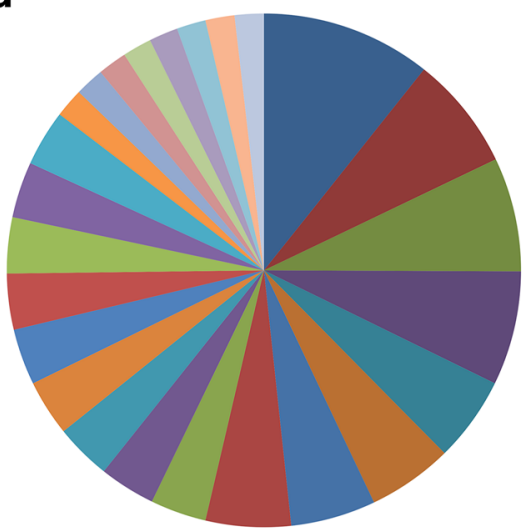

- Integrin signalling pathway (P00034)

Apoptosis signaling pathway (P00006)

- Inflammation mediated by chemokine and cytokine signaling pathway (P00031)

n Gonadotropin-releasing hormone receptor pathway (P06664)

- Ubiquitin proteasome pathway (P00060)

—FGF signaling pathway (P00021)

Fig. 2 Functional pathway analysis of mRNAs enriched in Lean- and MetS-EVs. a Lean-EVs contained genes encoding regulators of transcription, isopeptide bond, non-membrane-bounded organelle, and vesicle-mediated transport, among others. b Genes enriched in MetS-EVs were primarily involved in translation elongation and ribonucleoprotein complex. c The top six functional categories of genes enriched in Lean-EVs showed pathways related to gonadotropin-releasing hormone receptor (P06664), axon guidance mediated by netrin (P00009), apoptosis signaling (P00006), angiogenesis (P00005), Wnt signaling (P00057), and TGF- $\beta$ signaling (P00052). d The top six functional categories of genes enriched pathway in MetS-EVs included integrin signaling (P00034), apoptosis signaling (P00006), inflammation mediated by chemokine and cytokine signaling (P00031), ubiquitin proteasome pathway (P00060), and FGF signaling (P00021) 
Table 2 Insulin signaling-related mRNAs enriched in LeanEVs

\begin{tabular}{ll}
\hline Official gene symbol & Gene name \\
\hline KRT18 & Keratin-18 \\
DSP & Desmoplakin \\
ADCY10 & Adenylate cyclase-10 \\
RGS16 & Regulator of G protein signaling-16 \\
HMGA2 & High mobility group AT-hook-2 \\
AMPD1 & Adenosine monophosphate deaminase-1 \\
PTPRE & Protein tyrosine phosphatase, receptor type-E \\
S100A9 & S100 calcium binding protein-A9 \\
PPP1R3E & Protein phosphatase-1 regulatory subunit 3E \\
NTRK1 & Neurotrophic receptor tyrosine kinase-1 \\
HEY1 & Hes related family BHLH transcription factor with \\
& YRPW Motif1 \\
SYNGAP1 & Synaptic Ras GTPase activating protein-1 \\
PFKFB3 & 6-Phosphofructo-2-kinase/fructose-2,6-biphos- \\
& phatase-3 \\
RAB27A & RAB27A, member RAS oncogene family \\
TAF15 & TATA-box binding protein associated factor-15 \\
STXBP2 & Syntaxin binding protein-2 \\
PCBP2 & Poly(RC) binding protein-2 \\
STX3 & Syntaxin-3 \\
ACVR2B & Activin A receptor type 2B \\
H2AFX & H2A histone family member-X \\
BLVRB & Biliverdin reductase \\
\hline &
\end{tabular}

lack mRNAs related TGF- $\beta$ signaling. Furthermore, we found that co-culture with MetS-EVs increases renal tubular cell inflammation in-vitro. Therefore, these observations demonstrate that MetS alters the genetic cargo of MSC-derived EVs, which might interfere with their ability to repair damaged tissues.

MSCs constitute a promising approach for cell therapy, but their function might be altered in a cardiovascular disease milieu, compromising MSC function. For example, MSCs from patients with MetS and diabetes show increased oxidative stress and autophagy, and fail to differentiate in functional adipocytes [11, 29]. Likewise, MSCs isolated from horses with MetS show impaired viability, oxidative stress, and senescence [12]. In agreement, we have previously shown that adipose tissue-derived MSCs obtained from pigs with MetS show enhanced adipogenic and osteogenic propensity and senescence compared to their Lean counterparts, which was partly mediated by adipose tissue inflammation [15]. More recently, Saleh et al. [30] summarized findings from preclinical studies testing the efficacy of adipose tissuederived MSCs on obesity and MetS. They found promising beneficial effects of MSC transplantation on obesity and MetS, and conclude that paracrine effects of MSCs may constitute their most biologically significant role.
Table 3 Insulin signaling-related mRNAs enriched in MetSEVs

\begin{tabular}{ll}
\hline Official gene symbol & Gene name \\
\hline NR5A1 & Nuclear receptor subfamily-5 group A member-1 \\
MAFB & MAF BZIP transcription factor-B \\
CCNA2 & Cyclin A2 \\
SOD3 & Superoxide dismutase-3 \\
NPY1R & Neuropeptide Y receptor-Y1 \\
ITGA2 & Integrin subunit alpha-2 \\
FTL & Ferritin light chain \\
HIST1H1B & Histone cluster-1 H1 family member-B \\
DHX9 & DExH-box helicase-9 \\
PPP1CB & Protein phosphatase-1 catalytic subunit beta \\
HNRNPD & Heterogeneous nuclear ribonucleoprotein-D \\
HMGB1 & High mobility group box-1 \\
EIF5B & Eukaryotic translation initiation factor-5B \\
SLC15A1 ${ }^{\text {a }}$ & Solute carrier family 15 member-1 \\
PARL & Presenilin associated rhomboid like \\
PSMD14 & Proteasome 26S subunit, non-ATPase-14 \\
DHFR & Dihydrofolate reductase \\
EIF2S1 & Eukaryotic translation initiation factor-2 subunit \\
RECQL & alpha \\
\hline
\end{tabular}

a mRNAs also upregulated in MetS-MSCs compared to Lean-MSCs

The paracrine function of MSCs is partly mediated by releasing EVs. As therapeutic tools, EVs may have a superior safety profile compared to their parent cells [31], because unlike cells they can be safely stored without losing function. In previous studies in healthy pigs [8-10], we found that adipose tissue-derived MSCs release EVs that transport genes and proteins capable of modulating several cellular pathways, particularly high levels of TGF- $\beta$-related genes and pro-angiogenic genes. The current study extends our previous findings, and underscores the selective content of genes encoding regulators of transcription, TGF- $\beta$ signaling, and angiogenesis in Lean-EVs. TGF- $\beta$ is an anti-inflammatory factor that regulates cell growth, differentiation, and fibrosis, as well as regulatory T-cells. Among its signaling partners are growth/differentiation factor-7 (or bone morphogenetic protein-12), which stimulates MSC differentiation [32] and induces tenogenesis in bone-marrow MSCs [33], and Activin receptor type-2B (ACVR2B), a member of bone morphogenetic protein signaling involved in regulating adult bone mass [34]. Wnt signaling is also one of the top functional categories of genes enriched in Lean-EVs, and has a close link to TGF- $\beta$ signaling. They synergistically modulate embryonic development, fibrotic disease, and tumor progression through interactions among Smad, Axin, Dvl and $\beta$-catenin [35]. For example, Adenomatous 


\section{a mRNA excluded from mRNA excluded from}
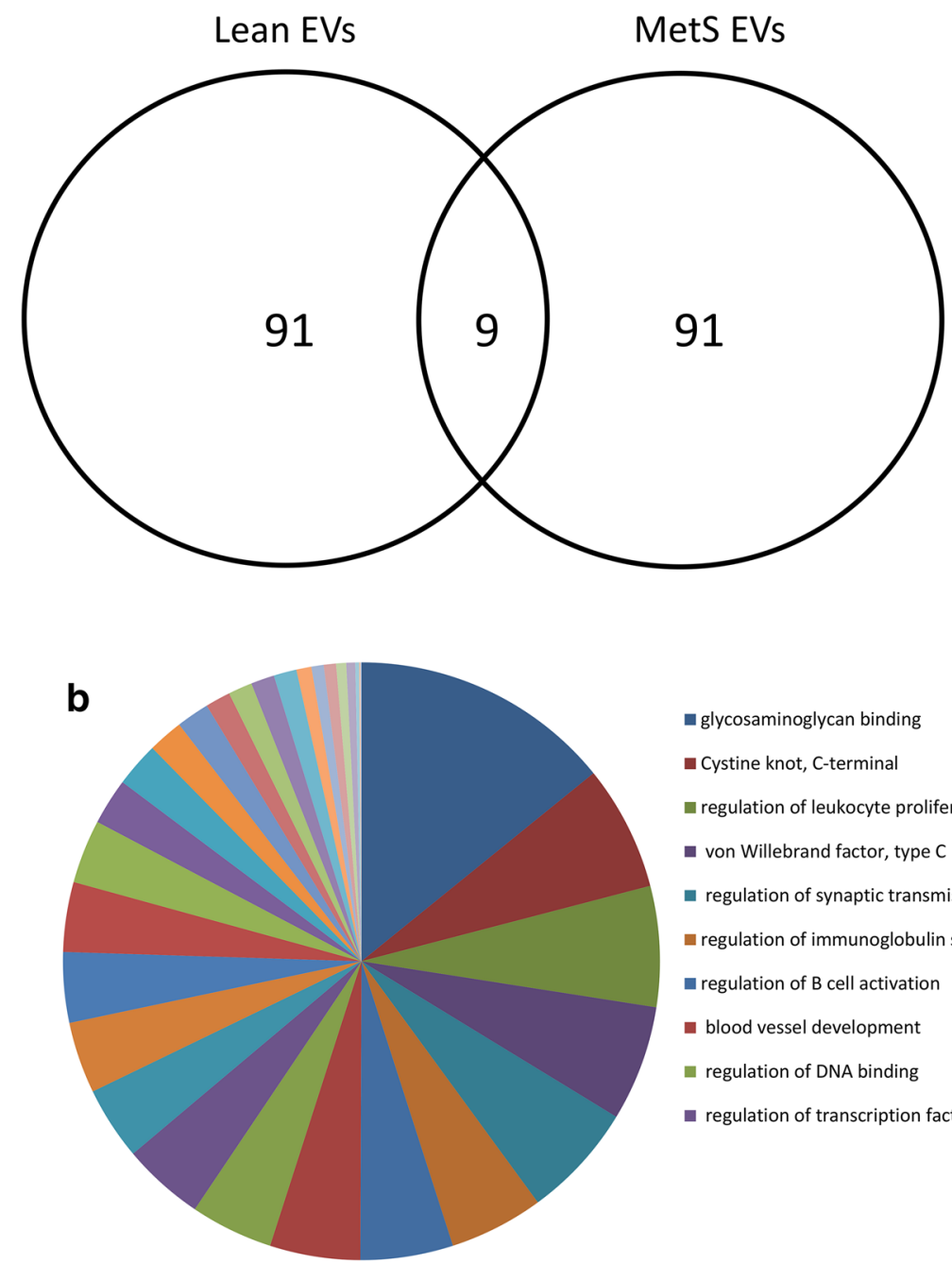

glycosaminoglycan binding

Cystine knot, C-terminal

regulation of leukocyte proliferation

- von Willebrand factor, type $C$

regulation of synaptic transmission

- regulation of immunoglobulin secretion

regulation of $\mathrm{B}$ cell activation

- blood vessel development

regulation of DNA binding

n regulation of transcription factor activity

C

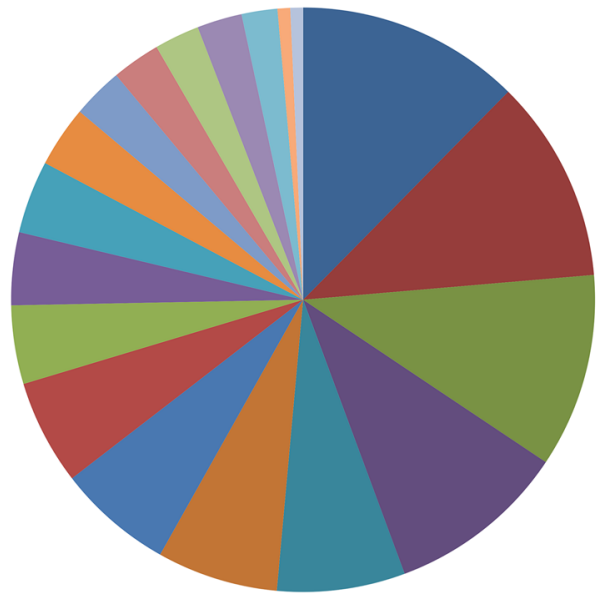

- TGFB

positive regulation of RNA metabolic process

a positive regulation of transcription

n carboxylic acid biosynthetic process

- cellular cation homeostasis

contractile fiber part

Evasculature development

- cellular homeostasis

Fig. 3 mRNAs excluded from Lean- and MetS-EVs. a Top 100 mRNAs excluded from Lean- and MetS- EVs revealed only nine common genes. Functional analysis of mRNAs showed selective genes excluded from Lean-EVs (b) and from MetS-EVs (c) 


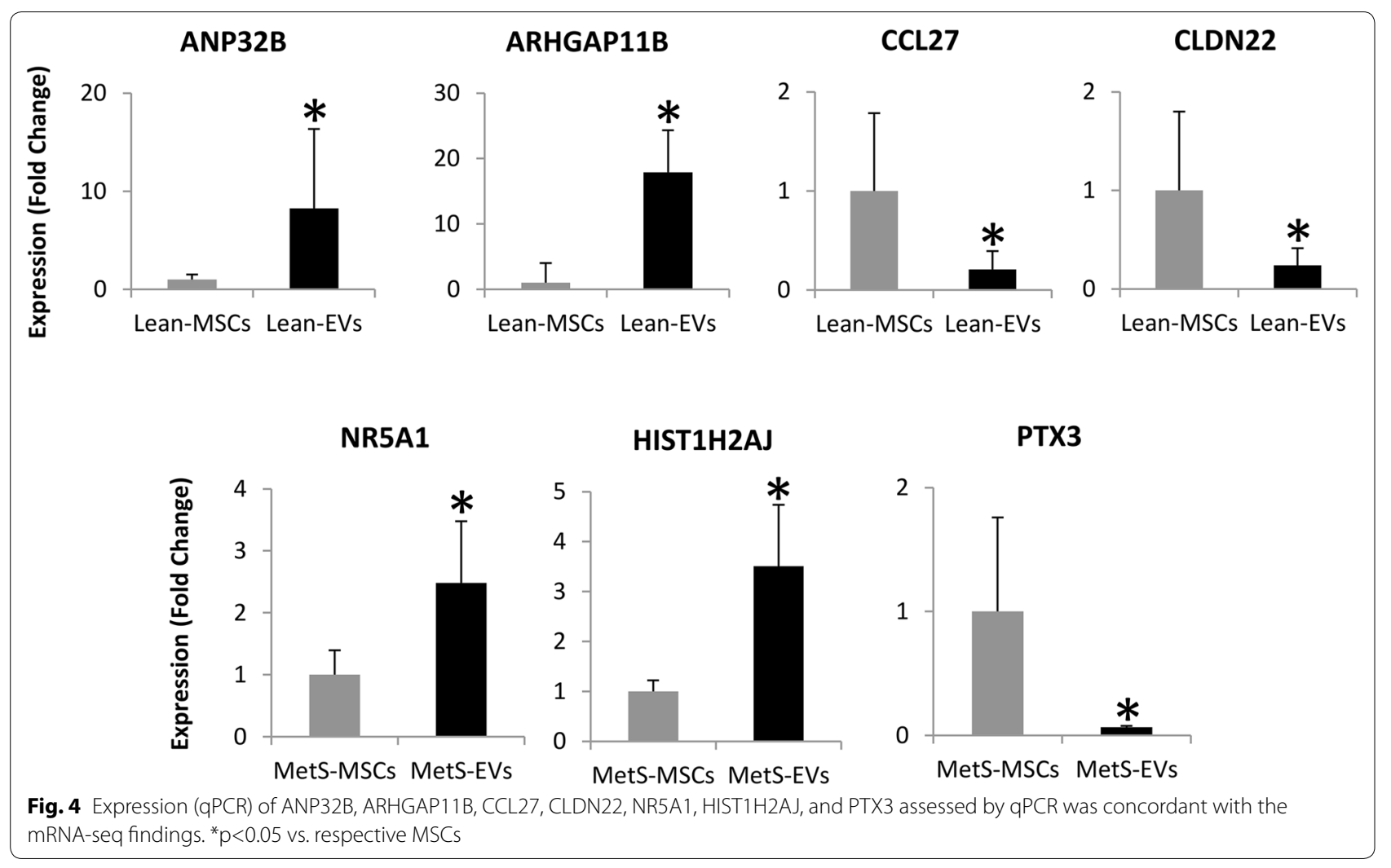

Polyposis Coli (Apc), enriched in Lean-EVs $(\times 1.5$ vs. MSCs), is a Wnt pathway regulator that in conjunction with TGF- $\beta$ regulates epithelial progenitor cell fate [36]. Hence, EVs may be endowed with functions linked to repair and regeneration of tissues and suppression of inflammation.

Compared to Lean-EVs, MetS EVs were found be enriched with mRNAs associated with inflammation, such as those encoding the integrin family proteins. Integrins are a family of transmembrane receptors that activate signal transduction pathways, inducing inflammation and early atherosclerosis. For example, ITGA2 (integrin alpha-2) triggers inflammation and endothelial dysfunction in patients with cardiovascular disease, chronic kidney disease, and type-2 diabetes [37-39], and joint inflammation in mouse models of rheumatoid arthritis [40]. Other pro-inflammatory genes enriched in MetS-EVs (Fig. 1c) included the phosphatidylinositol 4,5-bisphosphate 3-kinase catalytic subunit beta isoform (PIK3CB) [41] and several members of the ubiquitinproteasome pathway involved in development of inflammatory and autoimmune diseases [42]. For example, the ubiquitin conjugating enzyme E2-C (UBE2C), which activates several other genes (SLC2A1-CCNB2-HMMRKIF11-NUSAP1-PRC1-UBE2C), triggering inflammation [43], and the UCHL5 (or ubiquitin C-terminal hydrolase

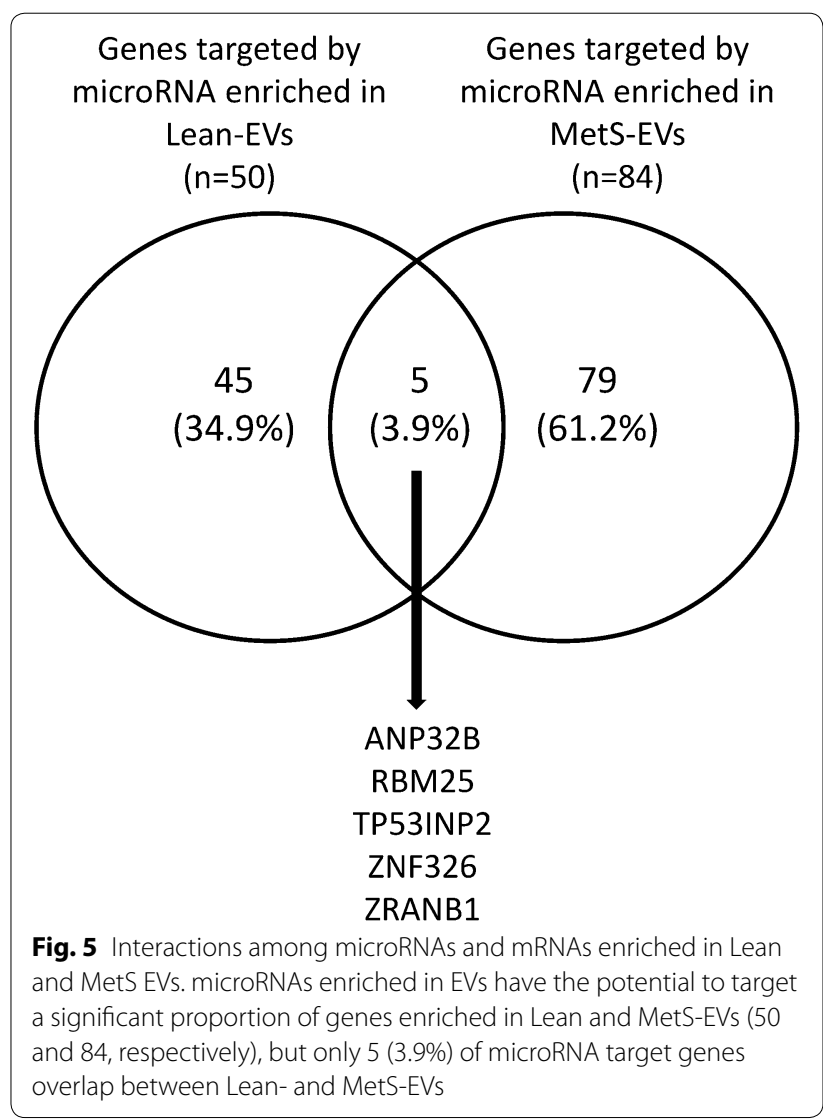


UCH37), which plays an important role in inflammation and host defense [44]. Lastly, mRNAs enriched in MetS-EVs encode proteins involved in FGF signaling, a family of proteins which can contribute to pathological conditions by modulating expression of inflammatory cytokines [45]. Hence, MetS-EVs have specific pro-inflammatory signatures that may impair the ability of MSCs to repair damaged tissues.

Genes that modulate apoptosis were similarly enriched in Lean- and MetS-EVs, but further analysis showed that those enriched in Lean-EVs encoded anti-apoptotic proteins, such as CFLAR (CASP8 and FADD-like apoptosis regulator) and heat-shock-related $70-\mathrm{kDa}$ protein-2 (HSPA2). CFLARL-deficient T-cells undergo severe cell death upon T-cell receptor stimulation, involving apoptosis and necroptosis [46]. Likewise, HSPA2 increases long-term survival of cells subjected to heat shock and proteasome inhibitors, and can be a part of a system protecting cells against cytotoxic stimuli inducing proteotoxic stress [47]. In contrast, apoptosis-related genes that were enriched in MetS-EVs promote autophagy and apoptosis, including EIF2S1 and MAP3K5. EIF2S1 is an ER stress sensor that induces mitophagy through ER stress activation due to reactive oxygen species (ROS) accumulation, and MAP3K5 (also known as apoptosis signal-regulating kinase-1; ASK1) is a key component of ROS-induced JNK and p38 activation that activates apoptosis.

Our analysis also identified several mRNAs related to insulin signaling enriched in EVS, which were different between Lean-EVs and MetS-EVs. Interestingly, two mRNAs enriched in MetS-EVs were also enriched in MetS-MSCs, as we observed in a recent study [25]. The nuclear receptor subfamily- 5 group-A member- 1 (NR5A1, or steroidogenic factor-1), which modulates the expression of insulin-like factor 3, relaxin-like factor [48], and the oligopeptide transporter PepT1 solute carrier family-15 member-1 (SLC15A1), which is highly regulated by insulin [49]. These observations suggest that MetS alters mRNA expression related to insulin signaling in adipose tissue-derived MSCs and their daughter EVs.

To test whether MetS-induced changes in the cargo of MSC-derived EVs impact their anti-inflammatory and immunomodulatory potential, we compared the effects of Lean- and MetS-EVs in M1-polarized macrophages and renal tubular epithelial cells in vitro. We have previously demonstrated the ability of porcine adipose tissue-derived MSCs to switch macrophages from a pro-inflammatory M1 phenotype to $\mathrm{M} 2$, a repair-linked phenotype [17]. In the current study, we found that
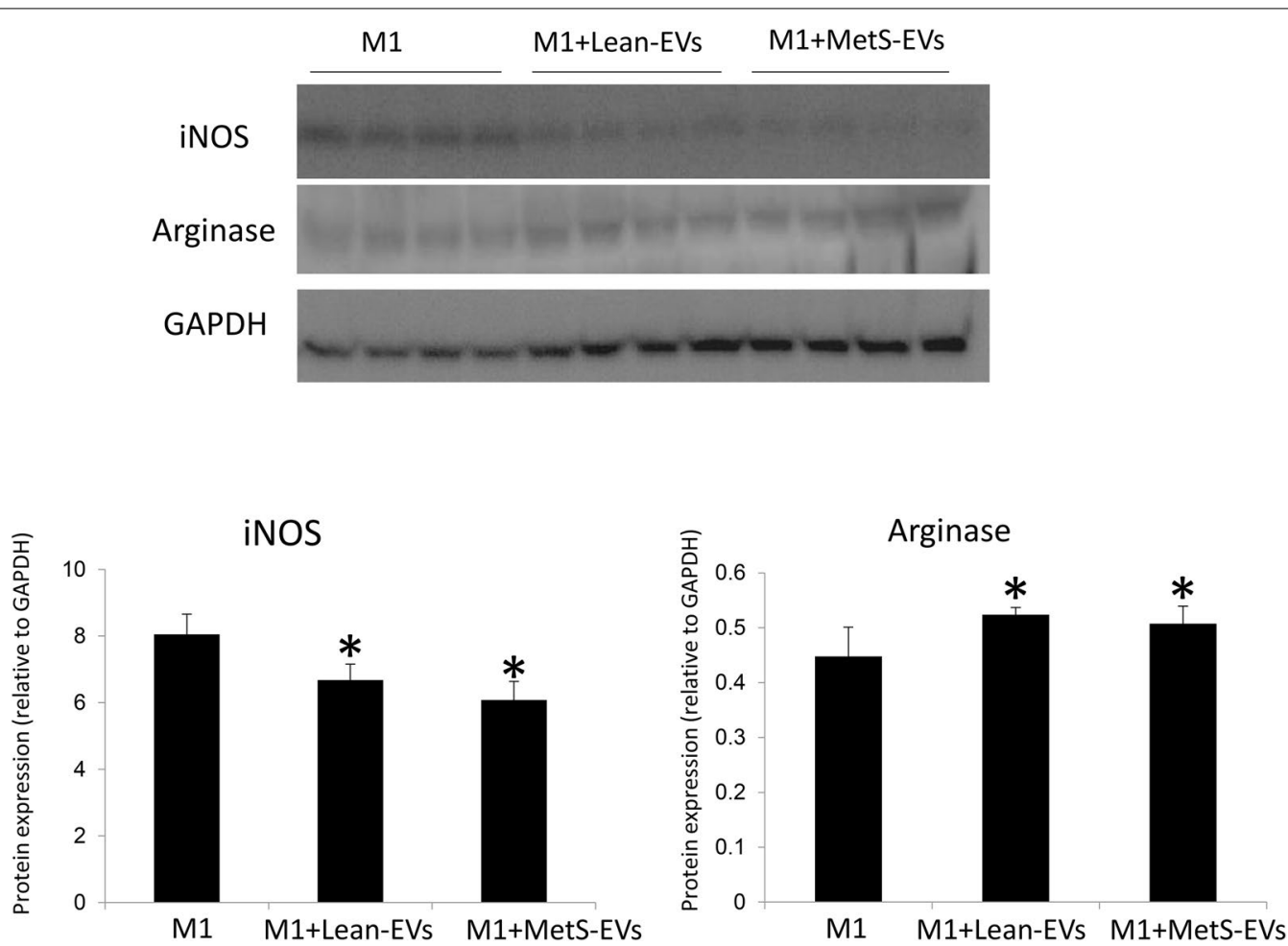

Fig. 6 MSC-derived EVs effects on macrophages. Co-culture with either Lean- or MetS-MSC-derived EVs similarly increased the expression of inducible nitric oxide synthase (iNOS) and decreased the expression of arginase-1 compared to M1-polarized macrophages, suggesting a switch to M2 phenotype. * $p<0.05$ vs. M1 
co-culture of M1-polarized macrophages with either Lean- or MetS-MSC-derived EVs similarly decreased the expression of the M1 markers and increased expression of M2 markers, in accordance with the similar capacity of Lean- and MetS-MSCs to restore an M2 phenotype in activated macrophages [15]. Contrarily, we found that expression of the pro-inflammatory cytokines TNF- $\alpha$ and MCP-1 was higher in renal tubular cells co-cultured with MetS-EVs compared to untreated cells and cells cocultured with Lean-EVs, consistent with our previous observation that TNF- $\alpha$ magnified MSC dysfunction in MetS [15]. Taken together, these observations reflect the complex regulation of inflammatory responses, and suggest that MetS-MSCs and their EVs induce pro-inflammatory cytokines, but do not directly alter macrophage phenotype.

These observations have important clinical implications. Autologous transplantation is preferred for MSC therapy in patients, many of who have comorbidities and cardiovascular risk factors. The primary mechanism of action of MSCs is the delivery of EVs carrying mRNAs, microRNAs, and proteins that are transferred to damaged cells. Thus, our observation that MetS induces changes in the mRNA cargo of MSC-derived EVs that affects their immunomodulatory properties in vitro suggests that the reparative capacity of these cells may be limited in subjects with MetS.

Our study has a number of strengths, including a novel swine model of MetS, the comprehensive characterization of the mRNA cargo of Lean- and MetS-EVs, and in vitro studies in macrophages and renal tubular cells to elucidate the impact of MetS-induced changes in the cargo of EVs on their immunomodulatory function. On the other hand, our study is limited by the modest number of animals and short duration of MetS. Nevertheless, MetS pigs developed several features of human MetS, and we detected a clear difference in the mRNA content of their EVs compared to their Lean counterparts. The

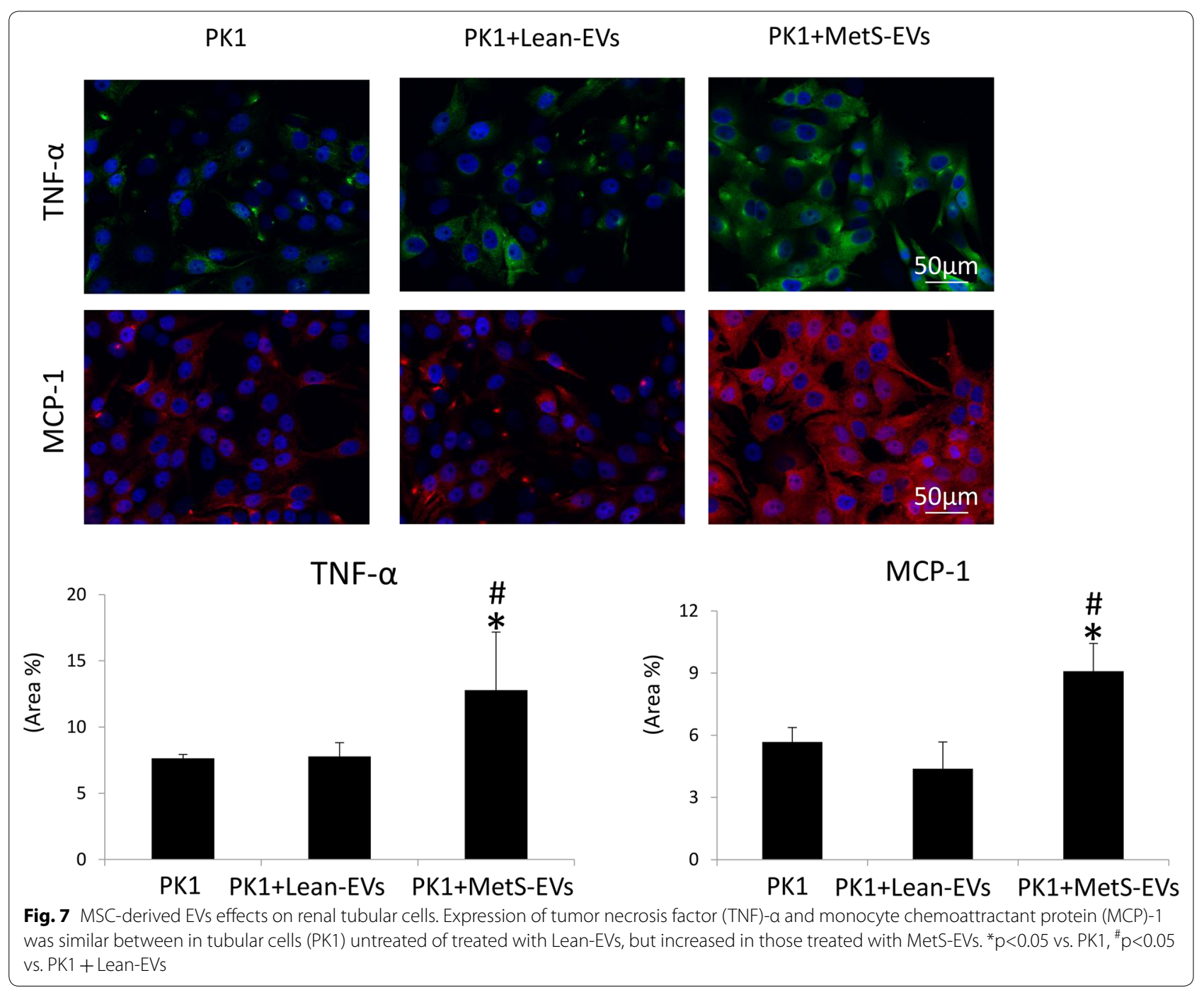


MSC mRNA profile can change with the passage of cells [50], thus we opted to study the 3rd passage cells that are relatively stable [51]. Additional studies are needed to explore the other functional ramification of the distinctive expression profile in MetS-EVs, and whether their mRNA content imposes a differential effect compared to Lean-EV on the phenotype of their recipient cells.

\section{Conclusions}

Using next-generation sequencing analysis, we discovered a distinct profile of mRNAs expressed in Lean- and MetSMSC-derived EVs. Genes enriched in Lean-EVs may reflect their predominant roles in regulation of transcription, TGF- $\beta$ signaling, and angiogenesis-associated proteins. In contrast, mRNAs enriched in MetS-EVs are primarily responsible for encoding pro-inflammatory proteins, and selectively depleted of proteins known to be implicated in the TGF- $\beta$ signaling pathway. Genes enriched in LeanEVs encode proteins involved in transcriptional regulation, whereas those packed in MetS-EVs modulate translation. Lastly, co-culture with either MetS-EVs increases renal tubular cell inflammation in-vitro. These findings indicate that MetS modulates the genetic cargo and possibly functionality of EVs, vectors of MSC inter-cellular communication. Although our mRNA-microRNA analysis suggests important post-transcriptional regulation, the underlying mechanisms and ramification of these differences warrant further investigation. These observations have important implications for cell-based therapy and may support development of strategies to improve the efficacy of MSCs and their EVs.

\section{Additional file}

Additional file 1. Individual mRNA expression levels obtained by RNAseq in Lean- and MetS-MSCs and their daughter EVs (Mvex) ( $n=5$ each). Expression values for each gene (Raw Counts) were normalized by the total number of reads per sample and corrected for gene length (reads per kilobasepair per million mapped reads, RPKM).

\footnotetext{
Abbreviations

MSCs: mesenchymal stem cells; EVs: extracellular vesicles; TGF: transforming growth factor; MetS: metabolic syndrome; RNA-seq: RNA sequencing; LDL: low-density lipoprotein; HOMA-IR: homeostasis model assessment of insulin resistance; RPKM: reads per kilobasepair per million mapped reads; qPCR: quantitative polymerase chain reaction; MEIS3: meishomeobox-3; HEY1: hairy/ enhancer-of-split related with YRPW motif-1; HSF5: heat-shock transcription factor family member-5; ACVR2B: Activin receptor type-2B; Apc: Adenomatous Polyposis Coli; EIF2S1: eukaryotic translation initiation factor-2 subunit-alpha; EIF5B: eukaryotic translation initiation factor 5B; RPS17: ribosomal protein S17; ITAG2: integrin alpha-2; PIK3CB: phosphatidylinositol 4,5-bisphosphate 3-kinase catalytic subunit beta isoform; UBE2C: ubiquitin conjugating enzyme E2-C; CFLAR: CASP8 and FADD-like apoptosis regulator; HSPA2: heat-shockrelated 70-kDa protein-2; ROS: reactive oxygen species; ASK1: apoptosis signalregulating kinase-1; NR5A1: nuclear receptor subfamily-5 group A member 1; SLC15A1: solute carrier family-15 member-1.
}

\section{Authors' contributions}

YM, AE: Collection and/or assembly of data, data analysis and interpretation, manuscript writing. XYZ, DRO: Collection and/or assembly of data, data analysis and interpretation. AL, AJV: Data analysis and interpretation, manuscript writing. LOL: conception and design, financial support, provision of study material, manuscript writing. All authors read and approved the final manuscript.

\section{Author details \\ 1 Divisions of Nephrology and Hypertension, Mayo Clinic, 200 First Street SW, Rochester, MN 55905, USA. ${ }^{2}$ Health Sciences Research \& Biomedical Statistics and Informatics, Mayo Clinic, Rochester, MN, USA. ${ }^{3}$ Cardiovascular Diseases, Mayo Clinic, Rochester, MN, USA. ${ }^{4}$ Orthopedic Surgery, Mayo Clinic, Rochester, MN, USA. ${ }^{5}$ Department of Nephrology, The First Hospital Affiliated to Jinan University, Guangzhou 510630, China.}

\section{Acknowledgements}

None.

\section{Competing interests}

The authors declare that they have no competing interests.

\section{Availability of data and materials}

The datasets containing all RNAseq data of the current study are available as Additional file 1.

\section{Consent for publication}

Not applicable.

\section{Ethics approval and consent to participate}

Animals were studied with the approval of the Institutional Animal Care and Use Committee (IACUC)

\section{Funding}

This study was partly supported by NIH Grant Numbers DK104273, HL123160, DK100081, DK102325, and DK106427.

\section{Publisher's Note}

Springer Nature remains neutral with regard to jurisdictional claims in published maps and institutional affiliations.

Received: 14 May 2018 Accepted: 16 July 2018

Published online: 21 July 2018

\section{References}

1. Dominici M, et al. Minimal criteria for defining multipotent mesenchymal stromal cells. The International Society for Cellular Therapy position statement. Cytotherapy. 2006;8(4):315-7.

2. Majka M, et al. Concise review: mesenchymal stem cells in cardiovascular regeneration: emerging research directions and clinical applications. Stem Cells Transl Med. 2017;6(10):1859-67.

3. Alagesan S, Griffin MD. Autologous and allogeneic mesenchymal stem cells in organ transplantation: what do we know about their safety and efficacy? Curr Opin Organ Transplant. 2014;19(1):65-72.

4. Nargesi AA, Lerman LO, Eirin A. Mesenchymal stem cell-derived extracellular vesicles for renal repair. Curr Gene Ther. 2017;17(1):29-42.

5. Nargesi AA, Lerman LO, Eirin A. Mesenchymal stem cell-derived extracellular vesicles for kidney repair: current status and looming challenges. Stem Cell Res Ther. 2017;8(1):273.

6. Eirin A, et al. Mesenchymal stem cell-derived extracellular vesicles attenuate kidney inflammation. Kidney Int. 2017;92(1):114-24.

7. Eirin A, et al. Mesenchymal stem cell-derived extracellular vesicles improve the renal microvasculature in metabolic renovascular disease in swine. Cell Transplant. 2018. https://doi.org/10.1177/0963689718780942.

8. Eirin A, et al. MicroRNA and mRNA cargo of extracellular vesicles from porcine adipose tissue-derived mesenchymal stem cells. Gene. 2014;551(1):55-64. 
9. Eirin A, et al. Glomerular hyperfiltration in obese African American hypertensive patients is associated with elevated urinary mitochondrial-DNA copy number. Am J Hypertens. 2017;30:1112-9.

10. Eirin A, et al. Comparative proteomic analysis of extracellular vesicles isolated from porcine adipose tissue-derived mesenchymal stem/stromal cells. Sci Rep. 2016;6:36120.

11. Kornicka K, Houston J, Marycz K. Dysfunction of mesenchymal stem cells isolated from metabolic syndrome and type 2 diabetic patients as result of oxidative stress and autophagy may limit their potential therapeutic use. Stem Cell Rev. 2018;14(3):337-45.

12. Marycz K, et al. Equine metabolic syndrome affects viability, senescence, and stress factors of equine adipose-derived mesenchymal stromal stem cells: new insight into EqASCs isolated from EMS horses in the context of their aging. Oxid Med Cell Longev. 2016;2016:4710326.

13. Meng $Y$, et al. Obesity-induced mitochondrial dysfunction in porcine adipose tissue-derived mesenchymal stem cells. J Cell Physiol. 2018;233(8):5926-36.

14. Pawar AS, et al. Adipose tissue remodeling in a novel domestic porcine model of diet-induced obesity. Obesity (Silver Spring). 2015;23(2):399-407.

15. Zhu XY, et al. Functional plasticity of adipose-derived stromal cells during development of obesity. Stem Cells Transl Med. 2016;5(7):893-900.

16. Eirin A, et al. Adipose tissue-derived mesenchymal stem cells improve revascularization outcomes to restore renal function in swine atherosclerotic renal artery stenosis. Stem Cells. 2012;30(5):1030-41.

17. Eirin $A$, et al. Renal vein cytokine release as an index of renal parenchymal inflammation in chronic experimental renal artery stenosis. Nephrol Dial Transplant. 2014;29(2):274-82

18. Meng Y, et al. The metabolic syndrome alters the miRNA signature of porcine adipose tissue-derived mesenchymal stem cells. Cytometry Part A. 2017:93:93-103.

19. Nargesi AA, Lerman LO, Eirin A. Mesenchymal stem cell-derived extracellular vesicles for renal repair. Curr Gene Ther. 2017;17:29-42.

20. Eirin A, et al. Integrated transcriptomic and proteomic analysis of the molecular cargo of extracellular vesicles derived from porcine adipose tissue-derived mesenchymal stem cells. PLoS ONE. 2017;12(3):e0174303.

21. Sun Z, et al. CAP-miRSeq: a comprehensive analysis pipeline for microRNA sequencing data. BMC Genomics. 2014;15:423.

22. Kalari KR, et al. MAP-RSeq: mayo analysis pipeline for RNA sequencing. BMC Bioinform. 2014;15:224

23. Robinson MD, McCarthy DJ, Smyth GK. edgeR: a bioconductor package for differential expression analysis of digital gene expression data. Bioinformatics. 2010;26(1):139-40.

24. Dudakovic A, et al. High-resolution molecular validation of self-renewal and spontaneous differentiation in clinical-grade adipose-tissue derived human mesenchymal stem cells. J Cell Biochem. 2014;115(10):1816-28.

25. Conley SM, et al. Metabolic syndrome alters expression of insulin signaling-related genes in swine mesenchymal stem cells. Gene. 2018;644:101-6.

26. Meng $\mathrm{Y}$, et al. The metabolic syndrome alters the miRNA signature of porcine adipose tissue-derived mesenchymal stem cells. Cytometry Part A. 2018;93(1):93-103.

27. Martinez FO, et al. Transcriptional profiling of the human monocyteto-macrophage differentiation and polarization: new molecules and patterns of gene expression. J Immunol. 2006;177(10):7303-11.

28. Zhu XY, et al. Mesenchymal stem cells and endothelial progenitor cells decrease renal injury in experimental swine renal artery stenosis through different mechanisms. Stem Cells. 2013;31(1):117-25.

29. Barbagallo I, et al. Diabetic human adipose tissue-derived mesenchymal stem cells fail to differentiate in functional adipocytes. Exp Biol Med (Maywood). 2017;242(10):1079-85.

30. Saleh F, et al. Adipose-derived mesenchymal stem cells in the treatment of obesity: a systematic review of longitudinal studies on preclinical evidence. Curr Stem Cell Res Ther. 2018;13(6):466-75.
31. Andaloussi SELA, et al. Extracellular vesicles: biology and emerging therapeutic opportunities. Nat Rev Drug Discov. 2013;12(5):347-57.

32. Shim EK, et al. Autogenous mesenchymal stem cells from the vertebral body enhance intervertebral disc regeneration by paracrine interaction: an in vitro pilot study. Cell Transplant. 2016;25:1819-32.

33. Otabe K, et al. Transcription factor Mohawk controls tenogenic differentiation of bone marrow mesenchymal stem cells in vitro and in vivo. J Orthop Res. 2015;33(1):1-8.

34. Kokabu S, et al. BMP3 suppresses osteoblast differentiation of bone marrow stromal cells via interaction with Acvr2b. Mol Endocrinol. 2012;26(1):87-94.

35. Rao C, et al. Crosstalk between canonical TGF-beta/Smad and Wnt/ beta-catenin signaling pathway. Zhejiang Da Xue Xue Bao Yi Xue Ban. 2013;42(5):591-6.

36. Li C, et al. Apc deficiency alters pulmonary epithelial cell fate and inhibits Nkx2.1 via triggering TGF-beta signaling. Dev Biol. 2013;378(1):13-24.

37. Wang $Y$, et al. Predictive role of multilocus genetic polymorphisms in cardiovascular disease and inflammation-related genes on chronic kidney disease in Type 2 diabetes - an 8-year prospective cohort analysis of 1163 patients. Nephrol Dial Transplant. 2012;27(1):190-6.

38. Luk AO, et al. Predictive role of polymorphisms in interleukin-5 receptor alpha-subunit, lipoprotein lipase, integrin A2 and nitric oxide synthase genes on ischemic stroke in type 2 diabetes - an 8-year prospective cohort analysis of 1327 Chinese patients. Atherosclerosis. 2011;215(1):130-5.

39. Yurdagul A Jr, et al. alpha5beta1 integrin signaling mediates oxidized low-density lipoprotein-induced inflammation and early atherosclerosis. Arterioscler Thromb Vasc Biol. 2014;34(7):1362-73.

40. Peters MA, et al. The loss of alpha2beta1 integrin suppresses joint inflammation and cartilage destruction in mouse models of rheumatoid arthritis. Arthritis Rheum. 2012:64(5):1359-68.

41. Huang JB, et al. Inhibition of the PI3K/AKT pathway reduces tumor necrosis factor-alpha production in the cellular response to wear particles in vitro. Artif Organs. 2013;37(3):298-307.

42. Wang J, Maldonado MA. The ubiquitin-proteasome system and its role in inflammatory and autoimmune diseases. Cell Mol Immunol. 2006;3(4):255-61.

43. Zhou H, et al. High EGFR_1 inside-out activated inflammation-induced motility through SLC2A1-CCNB2-HMMR-KIF1 1-NUSAP1-PRC1-UBE2C. J Cancer. 2015;6(6):519-24.

44. Mazumdar T, et al. Regulation of NF-kappaB activity and inducible nitric oxide synthase by regulatory particle non-ATPase subunit 13 (Rpn13). Proc Natl Acad Sci USA. 2010;107(31):13854-9.

45. Beenken A, Mohammadi M. The FGF family: biology, pathophysiology and therapy. Nat Rev Drug Discov. 2009;8(3):235-53.

46. He MX, He YW. CFLAR/C-FLIPL: a star in the autophagy, apoptosis and necroptosis alliance. Autophagy. 2013;9(5):791-3.

47. Filipczak PT, et al. HSPA2 overexpression protects V79 fibroblasts against bortezomib-induced apoptosis. Biochem Cell Biol. 2012;90(2):224-31.

48. Walther N, et al. Differentiation-specific action of orphan nuclear receptor NR5A1 (SF-1): transcriptional regulation in luteinizing bovine theca cells. Reprod Biol Endocrinol. 2006;4:64.

49. Der-Boghossian AH, et al. Role of insulin on jejunal PepT1 expression and function regulation in diabetic male and female rats. Can J Physiol Pharmacol. 2010;88(7):753-9.

50. Bonab MM, et al. Aging of mesenchymal stem cell in vitro. BMC Cell Biol. 2006:7:14.

51. Noer A, et al. Stable CpG hypomethylation of adipogenic promoters in freshly isolated, cultured, and differentiated mesenchymal stem cells from adipose tissue. Mol Biol Cell. 2006;17(8):3543-56. 